\title{
Quick Response Disaster Research: Opportunities and Challenges for a New Funding Program
}

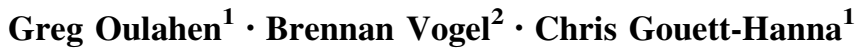

Accepted: 1 September 2020/Published online: 18 September 2020

(C) The Author(s) 2020

\begin{abstract}
Quick response research conducted by social scientists in the aftermath of a disaster can reveal important findings about hazards and their impacts on communities. Research to collect perishable data, or data that will change or be lost over time, immediately following disaster has been supported for decades by two programs in the United States, amassing a collection of quick response studies and an associated research culture. That culture is currently being challenged to better address power imbalances between researchers and disaster-affected participants. Until recently, Canada has not had a quick response grant program. In order to survey the state of knowledge and draw from it in helping to shape the new program in Canada, this article systematically analyzes the body of research created by the two US programs. The results reveal a wide-ranging literature: the studies are theoretically, conceptually, topically, and methodologically quite unique to one another. This diversity might appropriately reflect the nature of disasters, but the finding that many studies are not building on previous quick response research and other insights indicate opportunities for how a new grant program in Canada can contribute to growing a robust subdiscipline of disaster research.
\end{abstract}

Keywords Canada - Disaster research · Hazards · Quick response $\cdot$ Social science

Greg Oulahen

greg.oulahen@ryerson.ca

1 Department of Geography and Environmental Studies, Ryerson University, Toronto, ON M5B 2K3, Canada

2 Centre for Environment and Sustainability, Western University, London, ON N6A 3K7, Canada

\section{Introduction}

Post-disaster quick response social science research has the potential to uncover important findings that can advance understanding of hazards and their impacts on people and communities. Some of that information can be found in socalled "perishable" data, or data that will change or be lost over time, so it is critical for researchers to collect it from the site of the disaster while it still exists. These two central tenets have inspired and guided post-disaster quick response research over the past five decades in the United States, funded by long-running programs at the Disaster Research Center at University of Delaware and the Natural Hazards Center at University of Colorado Boulder. Until recently, Canada has not had a program to fund quick response disaster research by social scientists. With a long history of disasters affecting communities across the country, this gap has resulted in a limited number of quick response studies and associated research culture in Canada.

In an effort to take stock of the field and inform early decisions on the shape of a new quick response research program, this study collected and analyzed 94 completed quick response studies funded by the two US research programs. The findings of the analysis, set within the context of the literature on post-disaster research, provide insight to how quick response research is operationalized and how a new program might maximize its contribution to this field of study. The analysis is further supported by the first author's post-disaster experiences in urban and rural communities in Canada immediately following flood, wildfire, and tornado events, as well as early involvement in establishing the quick response research program. This program is emerging at a time of change in disaster research. Not only are many factors increasing disaster risk, the culture and ethics of disaster research are being 
challenged to address longstanding power imbalances between researchers and disaster-affected communities (Gaillard 2019; Gaillard and Peek 2019). The type of change being called for will make a profound difference in how disaster research is conducted and the findings it reveals. The compatibility of "quick" response research and the amount of time necessary to actually heed this call, however, presents challenges that disaster researchers and funders must consider.

This article aims to advance the field of study by conducting a critical, systematic analysis of quick response research, drawing insights from it to add to the discussion of how quick response research is best conducted and funded. It finds a wide-ranging literature: studies are theoretically, conceptually, topically, and methodologically quite unique to one another. While this diversity might appropriately reflect the nature of disasters, attention to some key aspects of doing quick response research can help to build this body of knowledge. After situating quick response research in context, the article describes the findings of the analysis and how the new program in Canada can address them.

\section{Quick Response Disaster Research}

Disasters are devastating to affected people and communities but provide research opportunities to better understand how to reduce future risk in that and other places. Quick or rapid response research to gather perishable data soon after a disaster can reveal important findings that would otherwise be lost as the recovery progresses (Quarantelli 1997; Michaels 2003; Stallings 2007). Perishable data that may be valuable to social scientists include, for example, observations of activities in context, individual accounts of the disaster or recovery activities, evolving plans and improvisation for facilities and personnel, and volunteer or nonofficial activities (Kendra and Gregory 2015). Findings from this data can make important contributions to the academic literature and risk reduction policy. The nature of post-disaster response and recovery can erode some social barriers and enable access to key stakeholders such as victims, officials, and organizations that may be inaccessible at other times. While there are prime opportunities for research, knowledge production, and applied learning to occur from post-disaster environments, there has been no comprehensive assessment of quick response research. Interdisciplinary approaches to disaster science and research are still evolving and lack scholarly agreement and consistency based on theory, best practices, and measurable indicators of research success (NRC 2006). Moreover, there has been no systematic assessment of post-disaster research methods (Witt and Lill
2018). Regarding building post-disaster quick response research theory, Kendra and Gregory (2015, p. 8) state: "There are only a few scholars that provide detailed and comprehensive research on post-disaster field methods. There are fewer studies that directly contribute to the knowledge base of best practices of reconnaissance field research relevant to the political, legal, administrative, and ethical challenges of today." These gaps provide opportunities for new research and methodological innovations in the post-disaster field research environment.

Post-disaster research and fieldwork can be classified and categorized as early reconnaissance (days to about two weeks), emergency response and early recovery (days to about three months), and short-term recovery (three months to about two years) (NRC 2006). Longer-term recovery and reconstruction research (two to about 10 years) and revisiting the disaster-impacted community to document longerterm changes (5 to 10 years) complete the post-disaster research temporal classifications. A typical timeline for conducting a post-disaster quick response research project (for example, early reconnaissance, emergency response, and early recovery) begins with scanning media accounts and often times limited, ambiguous information, followed by the rapid development of a research proposal that would include informed and poignant research questions and credible research budgets involving key partners (NRC 2006). Project preparation recommendations typically include reviewing relevant literature, completing research ethics protocol, organizing logistics associated with postdisaster fieldwork (travel, transport, food, accommodation, and so on), and critically liaising and gaining access to key stakeholders involved with organizations and facilities associated with post-disaster management and response as key steps in the post-disaster research response timeline.

There has been little uniformity of methods or approaches to post-disaster research but since the 1920s postdisaster research has been conducted predominantly by utilizing interviews (Phillips 2002; Stallings 2002). A recent review found that qualitative approaches of data collection commonly include literature review of academic, policy, and technical documents, and stakeholder interviews (Witt and Lill 2018). Field questionnaires and field observations are also commonly used in case study research for the collection of perishable data. While it is generally considered to be important to collect perishable data from the early stages of disasters, Killian (1956) and Stallings (2002) scrutinize the validity and generalizability of study results produced by incongruent and haphazard disaster research methods, questioning whether post-disaster research methodologies exemplify a distinct and unique entity in academic, interdisciplinary research inquiries. Killian (1956) identifies the theoretical and methodological challenges involved with post-disaster field 
studies and the difficulties associated with determining dependent and independent variables in ephemeral postdisaster environments. For example, research methods for sampling, selecting informants, securing interviews, recording and coding data, and presenting findings have long presented methodological challenges to disaster researchers. Stallings (2007) criticizes findings as often dependent on erratic variables such as research timing and subjective access to key stakeholders or selective postdisaster environments. Methodologically, this aspect of post-disaster research may compromise the objective capabilities to make reasonable generalizations, presenting potential validity challenges to this type of research. In arguing for the development of improved disaster theory, Alexander (1993, xvii) asserts, "[...] I hope that one day there will be a sufficiently large body of theory to permit us to inaugurate a new "interdisciplinary discipline" dedicated to the understanding of disastrous natural phenomena and their effects, and hence to the service of humanity."

Kendra and Gregory (2015) conducted a stakeholder workshop to discuss best practices, protocols, and methods for accessing and capturing perishable data with limited resources and for adapting research methods to the postdisaster environment. The workshop built from the premise that there is limited availability of information on postdisaster research methodologies and research designs, funding mechanisms, and the ethical norms inherent in post-disaster fieldwork, with further recognition that interdisciplinary collaborations confront institutional barriers, researcher's varying expectations, and different sociocultural perspectives on appropriate research ethics in disaster zones. Workshop participants also noted the critical issue of developing reliable funding and administration models for supporting and executing multidisciplinary post-disaster research capable of balancing scientific rigor with applicable research outputs based on sound methodological practices. Conducting a quick response project is a time limited, often ambiguous research activity in pursuit of ephemeral information, necessitating the rapid development of a research proposal that would include developing and vetting informed and poignant research questions, as well as credible research budgets involving key partners (Kendra and Gregory 2015). Conducting quick response research after disaster requires expedited review processes and advanced planning to obtain ethics approval (Mukherji et al. 2014). Kendra and Gregory (2015) note the crucial element of developing reliable funding and research administration models for executing multidisciplinary post-disaster research requires institutional support.

\subsection{Changing Culture and Ethics of Disaster Research}

Gaillard (2019) and Gaillard and Peek (2019) call for a fundamental change in disaster research to address the ethical dilemmas and power imbalances between researchers and participants. They argue that local researchers should conduct research on local disasters, or at least be involved in making key decisions about studies by foreign researchers. Ensuring that clarity in research purpose and accountable, ethical standards of informed research consent are upheld in post-disaster research practices should be prioritized alongside research questions. This inclusive approach proposes building on existing local expertise to identify research gaps and coproduce knowledge that benefits local participants and communities. Kendra and Wachtendorf (2020, p. 363) disagree, however, with the notion of a code of conduct for disaster research, arguing that "it would be likely to create a compliance morass out of all proportion to any ostensible harm." They contend that community priorities are difficult to discern and rarely singular, so privileging some local stakeholders could even serve to recreate the conditions that led to the disaster, and maintain that disseminating and refining best practices for research is the better approach. Through collaborative engagement with local populations though, researchers can identify which needs might be supported through targeted, nuanced research activities (Gaillard and Peek 2019). Attention to researcher-participant dynamics in the post-disaster environment becomes a necessary research objective. Such research relationships are not easily fabricated, as they can involve complex social factors such as developing trust, reciprocity, and established social networks, often inculcated through substantial investments over long periods of time. These neglected sociocultural aspects of conducting ethical disaster research stand in contrast to the urgency and timeliness imperatives that seem to have driven much of existing quick response research.

Killian (1956), Schein (1987), and Gomez and Hart (2013) all question whose needs are driving the inquiry process of gathering of nebulously defined "perishable" data in post-disaster research. Gaillard and Gomez (2015) raise ethical concerns about the "gold rush" attitude of disaster research and the intentions of academic inquiry in this realm. In response to the "wild west" of disaster research conduct, some countries such as New Zealand, the Philippines, Brazil, and Indonesia have sought to protect their citizens by restricting and regulating research activities to ensure that post-disaster studies are locally and culturally grounded with domestic collaborators rather than subjecting already vulnerable, disaster-affected populations to further anxiety-inducing experiences through 
interactions with foreign researchers (Gaillard and Peek 2019). Long-standing scholarly debates related to researcher-participant conduct and the roles and responsibilities of researchers in relation to positionality and ethical motivations apply in post-disaster research environments (Brun 2009; Calgaro 2015). Much of the research conducted in the aftermath of the 2004 Indian Ocean Tsunami, for example, was produced by an inundation of academics from foreign countries. This influx angered domestic researchers and fatigued local populations, but it also contributed knowledge to understanding tsunami risks and how they can be mitigated (Gaillard and Peek 2019). This ethical dilemma is at the center of disaster research; a research process that recognizes and gives power to affected populations produces better knowledge and prevents it from coming at such a high cost.

This approach requires researchers' sensitivity to risk and benefit of participation for survivors, the abilities of the research participants to make decisions, the importance of upholding research ethics, and the need for the informed consent of stakeholders participating in research activities. Methodological issues such as gaining social license for conducting research through informal networking, securing entry into communities, and gaining access to data from stakeholders require making community contacts and recruiting study participants while remaining sensitive to post-disaster social dynamics, cultural factors, and norms. This requires critical attention to the researcher's position in the field (for example, gender, age, ethnicity), which can present research challenges or opportunities for data collection, depending on a researcher's identity markers. In potentially difficult and foreign field environments, attentiveness to the research context requires disaster researchers to be capable of flexibly brokering multiple roles and identities in the field (Mukherji et al. 2014). Researchers should pay attention to "[...] literacy levels of the participants, psychological readiness of interviewees (i.e., reliving the disaster experience during interviews), time constraints and stress levels of participants engaged in recovery activities, benefits for the community, issues of class and power and common local etiquettes and norms" (Mukherji et al. 2014, p. 824). This view holds that local or domestic researchers are better positioned to understand and respect the rights of vulnerable disaster-affected people to recover without being subjected to foreign research investigation and academic objectification (Brun 2009). Jazeel (2007) similarly highlights these ethical questions pertaining to why and for whom knowledge and theory are produced, arguing for new insights and approaches regarding how scholars can bridge North-South divides and power relations through transformative research practices. To address this, in part, Kendra and Gregory (2015) suggest that ethical post-disaster researchers first must develop better situational awareness to develop relevant research questions preceding the deployment of an effective research team with a clear purpose. This integrally requires the participation of key partners operating locally on the ground. Upon the completion of fieldwork, researchers can show respect and reciprocity by sharing research data and findings through follow-up visits, "member checking" the data, and reporting with affected stakeholders (Mukherji et al. 2014; Kendra and Gregory 2015).

Regarding the importance of conducting disaster research with local collaborators, Zwi et al. (2006) characterize the post-disaster environment as a quagmire of disrupted social and infrastructural networks, limited resources, health and safety risks, and environmentally hazardous conditions where human rights abuses and intense competition for aid resources are grafted onto already existing socioeconomic vulnerabilities. The effects of marginalization and social exclusion on exhausted, disaster-affected populations can be deep. Hunt et al. (2016) highlight how disaster researchers may be naively engaging in unethical data collection activities that have negative consequences, including re-traumatizing vulnerable populations or seeking research participants at inappropriate times, logistically impeding response and recovery relief efforts, negatively contributing to rapidly changing social dynamics in post-disaster environments, and adding to post-disaster confusion. Practical and ethical challenges of post-disaster research such as competing for access to scarce resources in constrained post-disaster conditions while operating with external time pressures can serve to undermine genuine intentions to coproduce research and knowledge. There is a need for researchers and funders to re-explore and develop new pathways for how disaster risks are studied in ways that challenge many prevailing orthodoxies (Lavell and Maskrey 2014). Quick response researchers and funders have a responsibility to understand and address the ethical issues and power imbalances present in disaster research.

\subsection{State of Quick Response Research in Canada}

The Disaster Research Center at University of Delaware and the Natural Hazards Center at University of Colorado Boulder have funded post-disaster research in the United States for more than 50 and 30 years, respectively, but Canada did not have a quick response research program for social scientists studying disasters until recently. Hazards and disaster researchers in Canada have made significant contributions to the field, with early leaders including Ian Burton, Kenneth Hewitt, and Joseph Scanlon (for example, Burton et al. 1978; Hewitt 1983; Scanlon et al. 1985). Scanlon was later affiliated with the Disaster Research 
Center at University of Delaware. The Institute for Catastrophic Loss Reduction (ICLR), founded in 1999 by Paul Kovacs with support from the property and casualty insurance industry and affiliated with the University of Western Ontario, is Canada's longest running hazards research institute. ICLR established the first quick response research program in Canada in 2016 and the program has since funded several studies.

Launched after years of consideration, the timing was in response to the Fort McMurray wildfire, Canada's most expensive insured disaster. The fledgling program has issued several calls for proposals related to spring flooding and summer wildfire events but is open to receiving proposals for any type of disaster at any time. The federallyfunded Marine Environmental Observation, Prediction and Response (MEOPAR) Network of Centres of Excellence has also been involved in supporting a small number of studies on coastal flood disasters in conjunction with ICLR but the network will cease operations at the end of its current cycle in 2022. In the year following the launch of the program, ICLR and MEOPAR co-hosted a full-day workshop in Toronto, with 16 participants from universities, funding councils, and federal government agencies (including the first and second author). ICLR and MEOPAR later co-hosted a session at the Canadian Risk and Hazards Network (CRHNet) Symposium in Halifax. The session was well attended by emergency managers and other hazards practitioners and researchers from across the country. The workshop and conference session were both instrumental in identifying opportunities and challenges for advancing quick response research in Canada.

\section{Systematic Review of Completed Quick Response Reports}

Given the identified gaps in the literature, a baseline assessment of existing examples of quick response studies can enable a better understanding of the current state of quick response research. To this end, 94 reports were systematically reviewed from the available archives of the University of Delaware Disaster Research Center $(\mathrm{n}=16)$ and the Natural Hazards Center at the University of Colorado Boulder $(\mathrm{n}=78)$ during fall 2019. In conducting a review with a comparative focus on epistemological and methodological components of adaptation or risk reduction case studies, Bisaro et al. (2018) code data based on the typology of the research questions addressed, the data collection methods used, and the role of the researcher in the study. They also suggest that data collection methods can be analyzed with regards to qualitative aspects of study design, sample selection methods, the types of data collection methods used, as well as the role of external stakeholders involved with problem definition and data collection activities in case study research. Building on this analytical framework, quick response study reports from both institutions were collected and analyzed for a variety of quantitative and qualitative attributes, including the disciplinary orientation of the researchers conducting the study, the geographic location of the study, the types of hazards studied, the research methods used in the study, the research questions posed by the study, the key findings, and next steps or recommendations emerging from the study.

\subsection{Results and Analysis}

The results of the analysis for location, hazard type, data collection timeline, research methods, and referencing are described in this section. Some recurring issues with the reports are then noted. All of the reviewed projects were conducted by researchers at US universities, although researchers were permitted to undertake projects on disasters that occurred in international settings. In total, $69 \%$ of the studies were conducted within the United States. The remaining $31 \%$ of the studies were on disasters outside the United States or in US territories. These international sites were located in Canada, Mexico, Puerto Rico, Haiti, and various nations in South America, Asia, and Oceania. A significant portion of US-based studies were conducted in states bordering the Gulf of Mexico, most of which were based on hurricane disasters (Fig. 1). The states with the most studies were Florida, Texas, California, and New York. In total, $39 \%$ of all studies were in these four states.

The studies were then categorized by type of hazard, with nearly half (45\%) based on tropical storms (Fig. 2). Approximately $86 \%$ of the tropical storms in the reports were hurricanes impacting the southern United States and the Caribbean, with the other $14 \%$ being typhoons and cyclones in the Eastern Hemisphere. Of the hurricanes studied, most were focused on the major hurricane events of the last 15 years: Katrina, Sandy, Harvey, and Maria. By far the most reported on event was Hurricane Sandy, which accounted for $26 \%$ of all tropical storm reports alone, or approximately $12 \%$ of all 94 reports reviewed. The next three hazard types all share similar popularity in reports: flooding, earthquakes, and tornados. The reports classified as other include oil spills, volcano eruptions, infectious diseases, and winter storms.

An important factor in quick response research is the length of the time between the disaster occurring and researchers arriving to begin collecting data. This is a crucial dimension of the research as perishable data, especially from sources such as human memory, are not static and can change dramatically as time progresses. The quick response reports were sorted analytically based on the NRC (2006) post-disaster research classifications, 


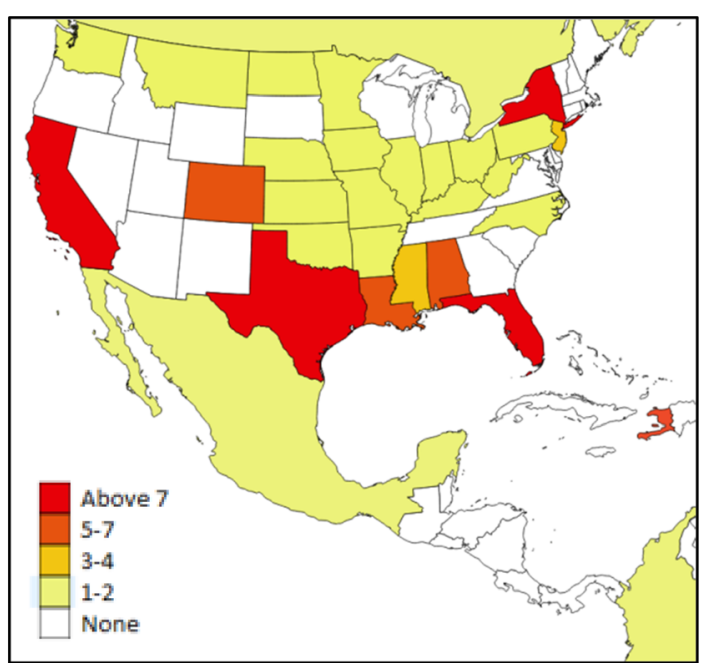

Fig. 1 Number of studies by location

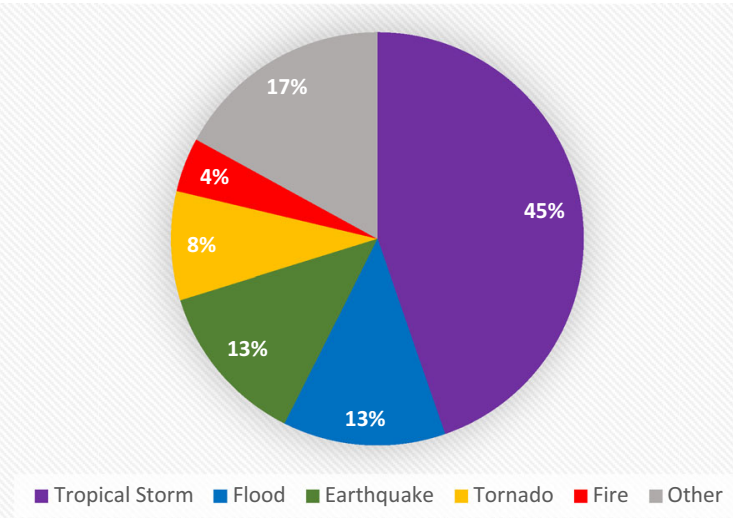

Fig. 2 Proportion of studies by hazard

including early reconnaissance (days to about 2 weeks), early recovery (up to 3 months), and short-term recovery (3 months to about 2 years) (Fig. 3). Of the reviewed studies, $28 \%$ did not include a time frame or date for the

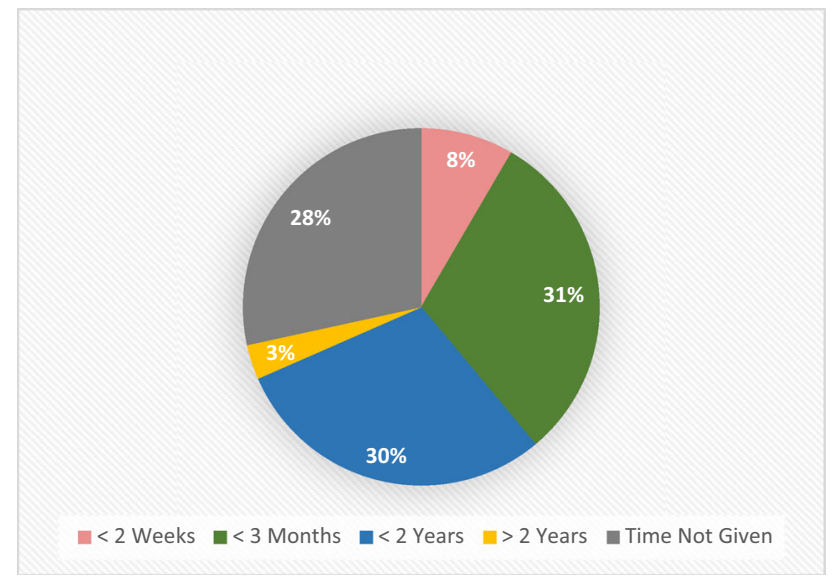

Fig. 3 Proportion of studies by data collection timing data collection phase. Only $8 \%$ of the projects were conducted in early reconnaissance phase following the disaster. Nearly one-third $(31 \%)$ of the studies occurred in the early recovery phase, while almost another third $(30 \%)$ occurred during short-term recovery. Finally, $3 \%$ of the reports conducted their data collection more than 2 years after the disaster.

The quick response studies used five distinct research methods: semistructured interviews, questionnaires, focus groups, field observations, and secondary document analysis (Fig. 4). Interviews were the most prevalent method employed for data collection, used by nearly half (46\%) of the studies. The main participants in the interviews were people impacted by the disaster, emergency responders, and policymakers. In total, $61 \%$ of the studies used a single research method and 39\% used multiple methods of data collection within the study.

To determine the extent to which the studies drew from or built upon other quick response research, the reports were cross-referenced. This was performed by querying the existence of the names of the authors of each quick response report within the pool of quick response reports. The results were checked to ensure that there were no false positives, and whether the papers referenced were quick response reports or later published versions of the same quick response research. The results showed that only 12 of the 94 total papers $(13 \%)$ referenced authors within the pool of quick response studies, either previous quick response studies or later published versions of the quick response papers. Four of those 12 papers were citing the author's own previous research. This finding indicates a lack of situating the study within the context of quick response research.

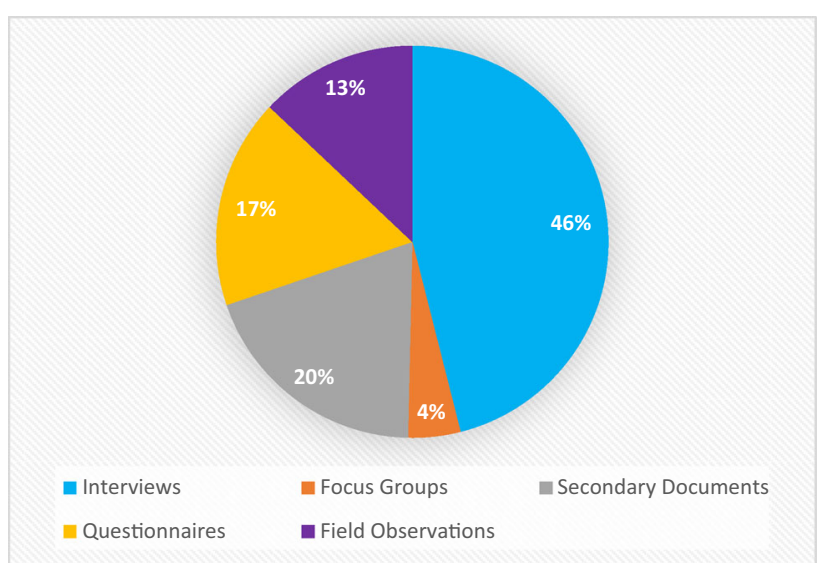

Fig. 4 Proportion of studies by research method 


\subsection{Recurrent Issues}

The analysis revealed some recurrent issues within the collection of studies, including the inconsistent length of reports, the lack of an explanation for needing a "quick" response, no description provided for the timing of the fieldwork, and no requirement for field research. Some reports are significantly shorter than the average report, with the shortest report less than one-third (29\%) the length of the average report. It is likely that some papers were overly brief due to time constraints for the researchers prior to the submission deadline. It is worth noting that some reports are more than 7000 words while others are less than 1500 words. Many of the reports do not describe the need for a "quick" response, leaving questions as to the importance of expediency for the study. Of the reports that provide the date range for data collection, many do not outline why it was a good time to conduct the research. As shown in Fig. 3, one-third (33\%) of the studies collected data more than three months after the disaster date. This proportion increases to nearly half (46\%) if the studies that do not provide a date are removed. Many researchers do not describe whether this timeline was optimal or problematic for the research. Some reports are focused on the recovery phase of the disaster process, but do not suggest whether the timing was in line with research objectives. A small minority of studies did not require field research, instead using data from secondary sources. This methodology was sometimes chosen due to issues encountered in the planning phase, while other studies sought data that did not require a presence in the field. Recognizing the difficult and complex nature of quick response research, these issues are noted here in the interest of reflecting on proposal and reporting guidelines.

\section{Discussion}

Despite decades of activity in post-disaster quick response social science research, there has been little systematic review or analysis of this body of work. This article seeks to address this gap by analyzing a collection of completed quick response study reports funded by two US research programs. The findings provide a baseline of the state of quick response research and insights that can be applied in establishing a new grant program in Canada. The main finding of this analysis is that quick response research does not constitute a subfield within disaster research by any factor other than the timing of the research. In addition to hazard and geographical variety, there is little theoretical, conceptual, or methodological consistency underpinning quick response research, making it a wide-ranging field of study. This finding raises the question of whether it would be constructive to work toward defining an explicitly quick response subdiscipline of disaster research and if such an approach would be useful to building knowledge. The new quick response grant program in Canada is well positioned to contribute to addressing this question.

Not being tied to a particular approach has the benefits of allowing for flexibility and openness, but may limit the field from creating knowledge by building on itself. Many of the studies state their theoretical approach and locate where they are coming from conceptually, but do not situate the research within the body of quick response research. The finding that the vast majority of reports do not cite previous quick response research indicates a lack of coherence and maturity in the field. By referring to the approaches, methods, or findings of completed quick response studies, researchers can actively contribute to building a subdiscipline of hazards research. Each study could contribute more to practice and theory by building from the knowledge gained in previous quick response studies. Diversity in approaches and methods can and should remain but there would be a foundation of knowledge from which to compare or contrast the findings of new research. Moreover, when new researchers enter the field in response to a major disaster there will be a clear and accessible body of knowledge for them to learn from and build upon in their research.

While it is the defining feature of the research, even the "quick" response timeframe is loosely interpreted (Michaels 2003). The studies are found to have been conducted at a wide range of times following the disaster. There are many good reasons for varied timing of postdisaster research but many of the reports are found to be unclear or missing key details about timelines of when the research was conducted and justification for that timing best addressing the research objectives. Other than a notion of sooner is better, they do not make the case for why it is important that the research be conducted at that time. Furthermore, many studies do not explicitly describe the perishability of the data collected, and why it would not exist or be different if the study was conducted at a later time. If the data is not, in fact, perishable then it negates the reason for rushing to collect it and imposing a researcher on a community struggling to recover from disaster. Given that data perishability is strongly influenced by changing post-disaster conditions, it is especially important for researchers to be clear about the timing of when the research was conducted. Participant interviews, found to be the primary research method used in quick response studies, seek rich and deep qualitative data that can inform the researcher's understanding of how time affects the data. Interviews may be sufficiently nuanced to provide context for ephemeral data and evolving participant perceptions. Lack of clarity around perishability of data and timing of 
the research are related and risk undermining the field. A subfield of quick response research could be better defined by clarifying conceptual links between the rationale that data is perishable and must be collected before it changes or disappears, and the idea that disasters are not exceptional events but rather embedded in the normal conditions of everyday life in the places they occur. The vulnerability approach has interrogated for more than four decades how these conditions turn hazards into disasters (Hewitt 1983; Wisner et al. 2004; Tierney 2014; Kelman et al. 2016). Studies may cite this foundational body of literature but many do not attempt to trace a chain of explanation to determine root causes of vulnerability. Identifying how key insights to understanding those root causes are indeed perishable would serve to further delineate quick response as a distinct subfield.

Quick response discourse has traditionally referred to researchers "deploying" to a disaster area. This language reflects a militaristic or command and control approach to responding to disaster. Such an approach may be at odds with a more critical approach to hazards research that seeks to understand root causes of vulnerability. Furthermore, it may perpetuate the "masculinist framing" of disaster research, wherein a researcher "from the outside" is the norm and emotional, affectual, and care-centered elements of disaster impacts and recovery are unexamined (AdamsHutcheson 2018, p. 149). That framing leads to and reinforces "drive by" research, wherein the researcher or team of researchers arrives quickly in a community and then leaves shortly after gathering sufficient data (Mukherji et al. 2014). "Helicoptering" into a place to interview or survey community members and then leaving as soon as the data is collected, never to return to report or member check the findings is clearly not in the best interest of disaster victims (Gaillard and Gomez 2015). The compatibility, though, between conducting research within a "quick" response timeframe and heeding the call to put first the local priorities of disaster-affected individuals and communities remains a difficult challenge. Whether it is possible to accomplish both objectives, and how to do it, is another area that should be addressed in establishing a quick response subdiscipline of disaster research.

A culture of quick response disaster research has existed for decades in the United States due to the two long-running funding programs. A similar research culture does not appear to exist in Canada. At the CRHNet special session, for example, some practitioners expressed a certain attitude of "ownership" over a local disaster and questioned why a researcher from another part of the country should travel there to do research and potentially get in the way of relief and recovery efforts. Those practitioners might have a well-meaning instinct that they are protecting residents and responders but this attitude in part reflects the lack of a quick response research culture in Canada as it exists in the United States, where researchers do not find that to be a major problem (Kendra and Gregory 2015). This attitude may pose a challenge for researchers entering a disaster area to conduct research rather than helping more directly with the recovery effort. But given the call to prioritize local needs in disaster research, it could be an opportunity for researchers to invite local practitioners and community members into leadership roles in the study. Incorporating local voices at the outset of efforts to grow a research culture in Canada can help to establish a norm in which community needs are integrated in research objectives.

There are other inherent challenges in doing post-disaster quick response research that must be recognized in establishing a research culture in Canada. One challenge is the difference between journalism and academic research. Most often journalists will beat researchers to the area, conduct interviews with victims and local officials, and report on it in the media. Public interest in the event will usually exist and it is the role of the media to provide information (Scanlon et al. 1985). The role of an academic researcher, to be clear, is quite different. A researcher must be conceptually sound and methodologically rigorous, have a reason for doing the study that is well supported by the literature and worth the time and energy of participants, and obtain research ethics approval before conducting any research with human subjects. Another challenge for the researcher is limited knowledge about the community before conducting the study, with only a preconceived idea about the place and perhaps an existing theoretical angle. Kendra and Gregory (2015) state that researchers should familiarize themselves with the community and local actors through mainstream and social media, but doing this quickly during the time between the disaster and arriving in the community will not lead to a deep understanding of the place.

A compounding challenge of doing quick response research is that it often does not synchronize well with the academic calendar. Its inherently responsive nature makes it difficult to plan for specific disasters, and preexisting commitments for researchers such as teaching, other ongoing projects, and administrative responsibilities can conflict with conducting fieldwork in another place. Graduate students must be trained, prepared, and free of other commitments like classes, teaching assistantships, and ready to travel for field research with short notice. Maintaining even a small group of graduate students who are ready and willing to do such work is a logistical challenge (Kendra and Gregory 2015). Obtaining an approved university research ethics protocol under a short time frame can be difficult, especially if the research ethics board is unfamiliar with quick response research (Hunt et al. 2016; Mezinska et al. 2016). These obstacles might 
be somewhat mitigated by planning ahead for hazards that have some degree of predictability, for example spring floods or summer wildfires. The location and timing of these events is unknown but some degree of planning for them is possible. Then when such a disaster inevitably occurs, the research team is prepared to focus a study on that particular event. Logistical challenges associated with quick response research should be recognized and mitigated by funders and researchers' institutions in the interest of enabling a research culture in Canada.

\section{Conclusion: Implications for a New Quick Response Research Program}

Quick response social science research can make valuable contributions to knowledge of disasters and how they affect communities but it must be undertaken carefully and purposefully if it is to contribute positively to both local populations and the academic literature. There are several key insights from this analysis that can contribute to the discussion around conducting and funding quick response research, particularly for developing a new quick response research program in Canada. Establishing a new program brings with it an opportunity to learn from and perhaps operate differently than existing programs. A new program does not have set routines or established procedures, and these can be created intentionally to maximize its contribution and minimize missteps or redundancy. The new quick response research program in Canada can serve to make dual contributions: better understanding hazards and disasters in Canada and their impact on people and communities, and building a robust subdiscipline of disaster research. Reflecting calls for local researchers to study local disasters, the program should focus on funding researchers at Canadian universities studying disasters in Canada. Canada is a large country by area but has a relatively small population, with a correspondingly low number of universities. The community of potential quick response researchers is likewise small, which could serve to benefit knowledge sharing and establishing a quick response research culture. Researchers should seek to involve local members of the community in their research, including researchers and practitioners, perhaps hiring students from that community as research assistants. Disasters affecting Indigenous communities should be studied by teams with members of those communities in leading roles.

To contribute to building a subdiscipline of disaster research, projects funded by the program should be required to address several identified needs. They should articulate the theoretical approach taken in the study, cite previous quick response studies that inform the research, identify the study as a quick response study and the timing of the research relative to the disaster, and make the case for why the data collected is in fact perishable. Making these components clear and explicit will enable future researchers to build from the studies and produce knowledge specifically related to quick response research. This does not mean studies must be standardized or all follow the same protocol; hazards researchers have recognized the pitfalls of such a prescriptive approach since critiques of the "dominant" paradigm emerged in the late 1970s. But being clear about important research decisions is not the same as following a standardized approach. Quick response researchers and funders are in positions to contribute to building an explicitly quick response subfield of study within disaster research, complete with specialized conceptual and methodological foundations. Such an effort is needed in order to be capable of addressing the current context of disaster impacts and changing expectations around the ethics of post-disaster research.

Acknowledgements Funding in support of this study was provided by the Marine Environmental Observation, Prediction and Response (MEOPAR) Network of Centres of Excellence of Canada. Paul Kovacs and Sophie Guilbault of the Institute for Catastrophic Loss Reduction have inspired and led much of the ongoing conversation around quick response research in Canada. Two anonymous reviewers and Editor Ilan Kelman made helpful comments that improved the manuscript.

Open Access This article is licensed under a Creative Commons Attribution 4.0 International License, which permits use, sharing, adaptation, distribution and reproduction in any medium or format, as long as you give appropriate credit to the original author(s) and the source, provide a link to the Creative Commons licence, and indicate if changes were made. The images or other third party material in this article are included in the article's Creative Commons licence, unless indicated otherwise in a credit line to the material. If material is not included in the article's Creative Commons licence and your intended use is not permitted by statutory regulation or exceeds the permitted use, you will need to obtain permission directly from the copyright holder. To view a copy of this licence, visit http://creativecommons. org/licenses/by/4.0/.

\section{References}

Adams-Hutcheson, G. 2018. Challenging the masculinist framing of disaster research. Gender, Place \& Culture 25(1): 149-153.

Alexander, D. 1993. Natural disasters. London: UCL Press.

Bisaro, A., M. Roggero, and S. Villamayer-Tomas. 2018. Institutional analysis in climate change adaptation research: A systematic literature review. Ecological Economics 151: 34-43.

Brun, C. 2009. A geographer's imperative? Research and action in the aftermath of disaster. The Geographical Journal 175(3): 196-207.

Burton, I., R.W. Kates, and G.F. White. 1978. The environment as hazard. New York: Oxford University Press.

Calgaro, E. 2015. If you are vulnerable and you know it raise your hand: Experiences from working in post-tsunami Thailand. Emotion, Space and Society 17: 45-54. 
Gaillard, J.C. 2019. Disaster studies inside out. Disasters 43(S1): S7S17.

Gaillard, J.C., and C. Gomez. 2015. Post-disaster research: Is there gold worth the rush? Jamba: Journal of Disaster Risk Studies 7(1): Article 120.

Gaillard, J.C., and L. Peek. 2019. Disaster-zone research needs a code of conduct. Nature 575: 440-442.

Gomez, C., and D.E. Hart. 2013. Disaster gold rushes, sophisms and academic neocolonialism: Comments on "Earthquake disasters and resilience in the global North". The Geographical Journal 179: 272-277.

Hewitt, K. 1983. The idea of calamity in a technocratic age. In Interpretations of calamity from the viewpoint of human ecology, ed. K. Hewitt, 3-32. Boston: Allen \& Unwin Inc.

Hunt, M., C.M. Tansey, J. Anderson, R.F. Boulanger, L. Eckenwiler, J. Pringle, and L. Schwartz. 2016. The challenge of timely, responsive and rigorous ethics review of disaster research: Views of research ethics committee members. PLoS ONE 11(6): Article e0157142.

Jazeel, T. 2007. Awkward geographies, spatializing academic responsibility, encountering Sri Lanka. Singapore Journal of Tropical Geography 28: 287-299.

Kelman, I., J.C. Gaillard, J. Lewis, and J. Mercer. 2016. Learning from the history of disaster vulnerability and resilience research and practice for climate change. Natural Hazards 82(S1): 129-143.

Kendra, J., and S. Gregory. 2015. Workshop on Deploying PostDisaster Quick-Response Reconnaissance Teams: Methods, Strategies, and Needs. University of Delaware Disaster Research Center Final Project Report No. 60. Newark, DE: University of Delaware.

Kendra, J., and T. Wachtendorf. 2020. Disaster-zone research: No need for a customized code of conduct. Nature 578: Article 363.

Killian, L.M. 1956. An introduction to methodological problems of field studies in disasters. Disaster Study Number 8, Committee on Disaster Studies. Washington, DC: National Academy of Science - National Research Council.

Lavell, A., and A. Maskrey. 2014. The future of disaster risk management. Environmental Hazards 13(4): 267-280.

Mezinska, S., P. Kakuk, G. Mijaljica, M. Waligora, and D.P. O'Mathuna. 2016. Research in disaster settings: A systematic qualitative review of ethical guidelines. BMC Medical Ethics 17: Article 62

Michaels, S. 2003. Perishable information, enduring insights? Undertaking quick response research. In Beyond September 11th: An account of post-disaster research. Special Publication No. 39, 15-48. Boulder, CO: Natural Hazards Research and Applications Information Center, University of Colorado.

Mukherji, A., N.E. Ganapati, and G. Rahill. 2014. Expecting the unexpected: Field research in post-disaster settings. Natural Hazards 73(2): 805-828.

NRC (National Research Council). 2006. Facing hazards and disasters: Understanding human dimensions. Washington, DC: National Academies Press.

Phillips, B. 2002. Qualitative methods and disaster research. In Methods of disaster research, ed. R.A. Stallings, 194-211. Newark, DE: International Research Committee on Disasters.

Quarantelli, E.L. 1997. The Disaster Center field studies of organized behavior in the crisis time period of disasters. International Journal of Mass Emergencies and Disasters 15(1): 47-69.

Scanlon, J., S. Alldred, A. Farrell, and A. Prawzick. 1985. Coping with the media in disasters: Some predictable problems. Public Administration Review 45: 123-133.

Schein, E.H. 1987. The clinical perspective in fieldwork. Qualitative Research Methods Series 5. Newbury Park: Sage.

Stallings, R.A. 2002. Methods of disaster research: Unique or not? In Methods of disaster research, ed. R.A. Stallings, 21-46. Newark, DE: International Research Committee on Disasters.

Stallings, R.A. 2007. Methodological issues. In Handbook of disaster research, ed. H. Rodriguez, E.L. Quarantelli, and R. Dynes, 55-82. New York: Springer.

Tierney, K. 2014. The social roots of risk: Producing disasters, promoting resilience. Stanford: Stanford University Press.

Wisner, B., P. Blaikie, T. Cannon, and I. Davis. 2004. At risk: Natural hazards, people's vulnerability, and disasters. New York: Routledge.

Witt, E., and I. Lill. 2018. Methodologies of contemporary disaster resilience research. Procedia Engineering 212: 970-977.

Zwi, A.B., N.J. Grove, C. Mackenzie, E. Pittaway, D. Zion, D. Silove, and D. Tarantola. 2006. Placing ethics at the centre, negotiating new spaces for ethical research in conflict situations. Global Public Health 1(3): 264-277. 